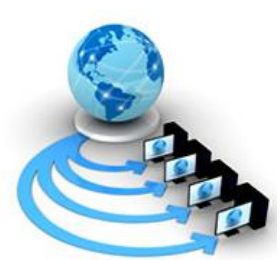

Volume 8, No. 9,November-December 2017

International Journal of Advanced Research in Computer Science

RESEARCH PAPER

Available Online at www.ijarcs.info

\title{
A PROPOSED PROTOTYPE OF E-PHARMACY WEB APPLICATION FOR THE CONSUMERS OF SAUDI ARABIA
}

\author{
Syed Asif Hassan \\ Department of Computer Science, Faculty of Computing \\ and Information Technology Rabigh (FCITR) \\ King Abdulaziz University, \\ Jeddah, Saudi Arabia
}

\author{
Tabrej Khan \\ Department of Information Sciences, Faculty of Computing \\ and Information Technology Rabigh (FCITR) \\ King Abdulaziz University, \\ Jeddah, Saudi Arabia
}

\begin{abstract}
E-commerce in recent years have achieved huge success by providing a virtual platform to do business with great ease. In this regard, we developed an electronic portal to buy and sell medicine online to encourage the concept of e-pharmacy in the Kingdom of Saudi Arabia. Saudi Arabia has a rich heritage traditional herbal medicine leading to the mushrooming of many regional pharmaceutical industries across the kingdom. However, the application of e-commerce principles in pharmacy has not been employed in Saudi Arabia. In this context, our proposed e-pharmacy portal will be first of its kind to provide customer and suppliers across the kingdom the benefits of E-pharmacy. Based on the results of the feedback from customers, retailers and suppliers across the kingdom following sustainable goals were identified: 1) the e-pharmacy web portal will allow transactions based on valid prescription, 2) the portal will allow retailer to upload their medicine and sell them online, 3) the portal will allow the customers to avail discounts on specific medicines and do payment through PayPal a trustworthy portal for performing online money transactions. A relational database will be created and managed using MySQL and PHP a server-side scripting language will be used to build the e-pharmacy web portal. The e-pharmacy practices will provide a stepping stone for the growth of online pharmacy in the kingdom.
\end{abstract}

Keywords:e-pharmacy portal, MySQL, PHP, E-commerce, prescription-based transactions

\section{INTRODUCTION}

In recent years, thekingdom has seen an increasing population of young Saudi's getting familiar with internet and internet-based applications. As a result of more and more Saudi's gaining interest in theinternet, the electronic commerce in Saudi Arabia is projected to reach approximately 6 billion by 2017. An estimated 70 million website is visited in a month and the percentage of people using theinternet in Saudi Arabia is increasing at the rate of 9.3 percent per year. As per recent surveys, souq.com is one of the largest e-commerce based enterprises in the kingdom with a market share of $13 \%$. Moreover, other e-commerce giants namely sukar.com with 8 $\%$, Namshi with 7 percent, and MarkaVIP Fashion with an overall market share of $5 \%$ contributetowards an estimated ecommerce market of $\$ 1.7$ billion making it one of the largest segment of e-commerce in Saudi Arabia [1].

In another survey conducted by MasterCard in 2014, it was found that a steady but consistent growth of e-commerce setup in the kingdom is because of reliable and trustworthy online payment system namely PayPal, CashU and credit card. Another noticeable findingof the survey was that in spite of using theforeign site for online shopping, the Saudi's prefer to do the same using local e-portal. The studies also show that kingdom has the necessary foundations for successful startups in E-commerce[2].

In this regard, our proposed e-pharmacy portal will provide a single platform for the suppliers and retailer to sell their medicine online to a wide range of customers across the kingdom. Our proposed portal i.e., www.saudiepharmacy.com will be one of its kind in the Kingdom of Saudi Arabia(KSA) where customers can visit the website (e-pharmacy web portal) and search medicines of their desired category andmanufacturer. Therefore, it will become very easy for the customer to select the medicinal products without wasting their time and money. Since the portal usestheprescription based purchase of medicine, therefore,the creation of e-pharmacy portal and e-prescription based practices will increase the safety and improve the quality of services provided to the customers across KSA. The present study is based on a successful running e-pharmacy model from India as well as epharmacy based studies done by researchers in the past [3-4]. Normally, in India where medicine is sold primarily at brickand-mortarpharmacies, selling medicine online by Netmed (https://www.netmeds.com/) was recognized as India's most pathbreaking start-up company and was awarded the prestigious NDTV unicorn start-up award for the year 2016. Therefore considering the advantages and the impact it will have on customers of Saudi Arabia we decided to build this eportal. Our proposed e-pharmacy online portal will provide the following advantages to the consumers namely; 1) Information: details of the medicinal product are available online; 2) Price and selection: Maximum Retail Price (MRP) of each medicine is uploaded which makes it easy for the customers to select; 3 ) Transportation: The customers receive the order in minimum working days within the kingdom of Saudi Arabia; 4) Convenience and safety: Easy to purchase and e-prescription will not allow any supply of medicine without valid eprescription. The current research paper is structured indifferent sections in the following way. Section II Describe the materialand method used to develop the e-pharmacy online portal. Section III explains the system analysis and design of the e- pharmacy portal and Section IV provide the concluding statementsabout the developed e-pharmacy portal.

\section{MATERIALS AND METHOD}

\section{A. Survey and data collection}

The questioner for the survey was prepared using a template of Google Forms (https://docs.google.com/forms/u/0/?tgif=d). 
The aim of the survey was the following: 1) to identify the need and requirements to develop an e-pharmacy application for buying and selling medicine online.; 2) to know the inclination of the consumers and sellers towards online pharmacy application. In order to perform the survey a Facebook group comprising of both the retailers selling medicine offline in Saudi Arabia and students as well as faculties of the Faculty of Computing and Information Technology, Rabigh (FCITR), King Abdulaziz University of SaudiArabia. The Google survey form with thebelow-pasted link was posted on the created Facebook group timeline and the comprising group members were requested to fill the survey form for review and further analysis.

https://docs.google.com/forms/d/e/1FAIpQLSfP7o-

Ju_wUQD3Hylc0Sssp43TSVnHiOmPNleT_IbwYp_D_ag/vie wform

The result of the questioner will be processed by the Google drive in the form of graphs for further analysis. The data generated from the survey will be helpful in designing and implementing an e-pharmacy application for buying and selling medicine online.

\section{B. Architecture of e-pharmacy online portal}

The present work is based on the principles of e-commerce [5-6]. An e-commerce based approach for selling medicine online was developed and was called as "Saudi-e-pharmacy". Since the project was of short duration and the requirement and technology for the implementation of theprojectwere very clear and fixed the waterfall model was utilized for developing the epharmacy application [7]. The phases in waterfall model are non-overlapping i.e., one phase is completed before starting the next phase. The linear-sequential life cycle of the applicationmodel consisted of five phases namely 1) Data collection (survey) for requirement analysis, 2) Designing the interaction framework of the application, 3) implementation interface and building the internal structure or database of the application using PHP (programming language) and MySQL server, 4) validation and testing the application using white-box and black-box, 5) deployment of thesystemand maintenance.

Unified Modeling Language (UML) was employedin the initial stages of system designing,to visualize the design of thee-pharmacy application [8]. The web application and database of e-pharmacy was developed using the Model-viewcontroller (MVC) in the implementation phase. The MVC architecture consists of three interlinked components. This division of components is done to segregate the inner representation of data from the ways data is accepted from and offeredat the user interface for the user to visualize and analyze [9]. The component model of MVC architecture uses MySQL as an open source relational database management system (RDMS) to manage the logic, rule, and data of thee-pharmacy application [10]. Secondly, the component view of MVC uses Hypertext Markup Language (HTML) a markup language to develop an interactive web portal of e-pharmacy [11]. The input data of the customers are processed into information for theuser to view. Finally, the component controller of MVC receives input from the user and subsequently interact withthe component view or model using a server-side scripting language i.e., Hypertext Preprocessor(PHP) [12]. Black box [13] and white box [14] was used to test the functional validity of modules involved in the development of theonline epharmacy application.

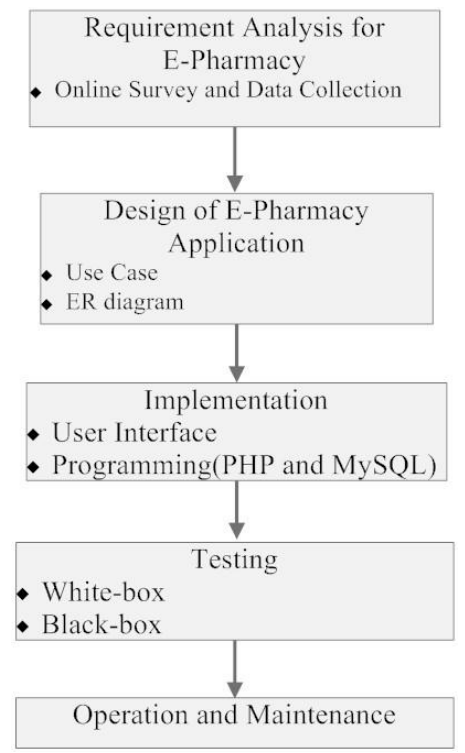

Fig. 1. Flow diagram depicting the steps involved in building the e-pharmacy portal

\section{RESULTS AND DISCUSSION}

\section{A. e-pharmacy application requirement}

The functional requirement ofthepharmacy application was based on the result of the questioner. The result of the questioner is graphically represented using aPie chart as shown in Fig. 2. The result of each questioner and their corresponding interpretation are as follow: 1) There were $36 \%$ female and 64 $\%$ male respondent; 2 ) There are $74.4 \%$ of the respondent who uses theinternet regularly with more than $10 \mathrm{hrs}$ per week. This data will give us an insight on the use of theinternet by the people of Saudi Arabia; 3) for the third question the respondentswere allowed for multiple answers. In this case, $66.7 \%$ of the respondent use internet for the purchase of goods and services information. While $23.1 \%$ of the respondent use the internet for information and $7.7 \%$ and $2.6 \%$ use the internet for downloading games and song and playing games, respectively. This data provides a clue regarding the activity of respondents when they are online; 4) the next data depicts that around $69.2 \%$ of the respondents use both computer andsmartphoneto accesstheinternet.while $25.6 \%$ of the respondent use asmartphone and the remaining $5.1 \%$ access internet via computers. This data shows us that the majority of respondent use both smartphone and computer therefore according to the data the application (e-pharmacy portal) should be accessible through smartphone as well as computers; 5) From the answers of thefifthquestion it appears that most of the respondent are buyer i.e., $74.4 \%$, while $20.5 \%$ are sellers and the remaining $5.1 \%$ are both seller and buyer; 6) The sixth question is targeting the respondent who is seller. The data represents that around $87.2 \%$ of their customers are from the kingdom. While the remaining $12.8 \%$ are from overseas. This data shows that a selling medicinal product online will be successful since majority of the customers of the retailer are from Saudi Arabia;

7) The next data inform us that $97.4 \%$ of the respondent are in favor of having an e-pharmacy application on web to buy and sell medicine online, while $2.6 \%$ of the respondent prefer retail shop for buying medicines;8) From this data it appears that the majority of the respondent i.e., $59 \%$ prefer online monetary transaction via credit card. While $20.5 \%$ prefers making payment via PayPal and $17.9 \%$, preferto pay cash on 
delivery since they don't consider online transaction reliable and trustworthy and lastly, $10 \%$ use debit card as a mode for doing online transactions. Based on the results of the survey the functional requirement of the application was set and the epharmacy prototype was designed.

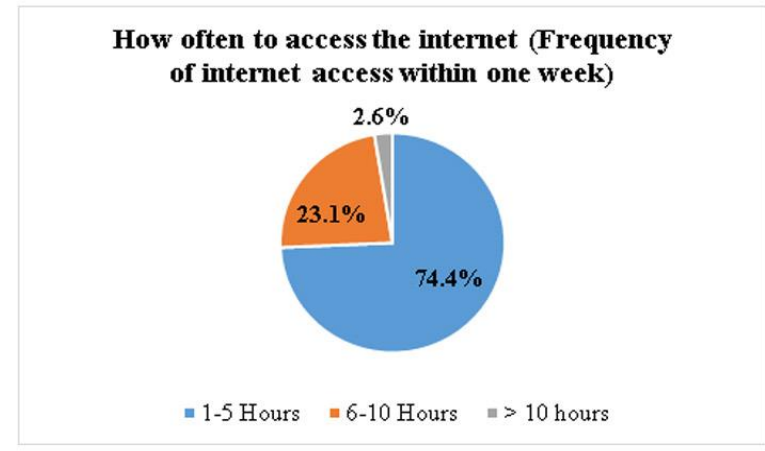

$=$ Male $=$ Female

The importance of accessing the internet

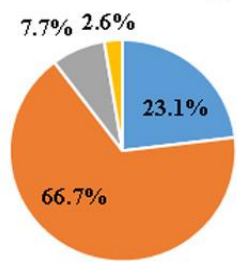

- Searching for information $=$ Purchase of goods orservices

" Download music/software = Playing games

Your current profession as

$5.1 \%$

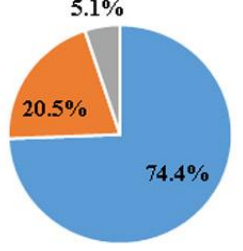

" Buyer $=$ Seller $=$ Both

Your preferred online payment system



- Cash on Delivery $=$ PayPal $=$ Credit Card $=$ Debit Card

Fig 2: Pie chart representing the results obtained for each questionnaire used in the survey 




Fig 3: UML diagram representing the relationship between the application and its stakeholders (User, admin, supplier)

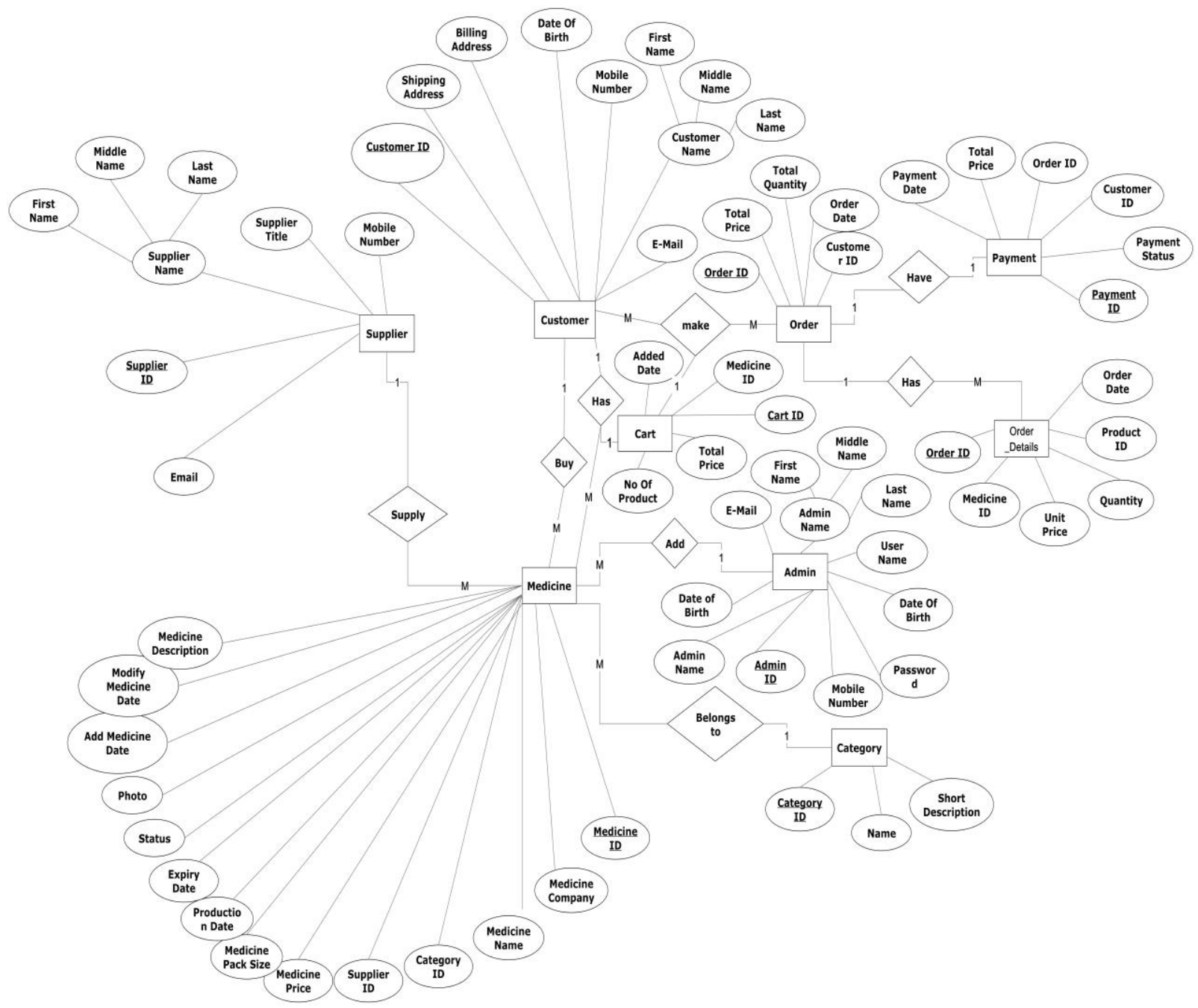

Fig 4: Entity relationship diagramof e-pharmacy application 


\section{B. e-pharmacy application design}

\section{1) Use case diagram for e-pharmacy application}

The functional requirement of the application was centered on the interaction of the stakeholder i.e., medicine seller, customers, suppliers and the proposed e-pharmacy online application. In this regard, the use case diagramwas used to model the action typically describing the overall interactions between the system (e-pharmacy application) and the stakeholders namely admin, user and medicine supplier as represented in Fig 3.

There are three actors namely user, admin and supplier and 20 use cases in the use diagram of the e-pharmacy application. The actor admin of e-pharmacy application after successful login, can manage medicine order details(edit/update/delete/view), manage medicine details (add/update/delete/help), manage customer detail (edit/delete/view), manage medicine supplier details (edit/add/delete/view) and finally manage customer's payments details (edit/ update/delete/view). Additionally, the actor user after successful login can manage personal information (view, change thepassword, change personal information, change contact information) also he can manage order (cancel, view) and can manage shopping cart (buy anitem, delete item view selected item).Finally, the supplier can view the order, theaddress of the customer and contact the transporter to send the order.

\section{2) ER diagram for e-pharmacy application}

As shown in Fig. 4 the ER diagram of the e-pharmacy application consists of 8 entities and they are 1) medicine, 2) customer, 3) supplier4) admin, 5) category of medicine, 6) order,7) order details, 8) payment. Each and every entity in the ER diagram hasspecificattributes based on their function and characteristics in the e-pharmacy application.

\section{C. e-pharmacy application implementation}

1) e-pharmacy application home page.

Aninteractive, user-friendlyinterface of e-pharmacy web portal was built for the customers to choose different category and brand of medicines by using a valid prescription.

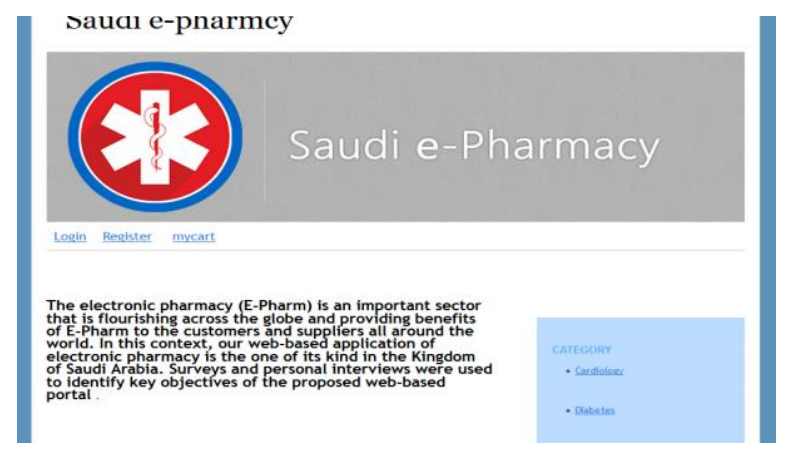

Fig 5: Saudi e-pharmacy user interface.

As shown in Fig. 5 the home page of the electronic portal consists of a header which comprises of a navigation bar consisting of 1) login, 2) register, 3) my cart. The body of the home page of e-pharmacy consist of 1) description about the epharmacy portal, 2) right menu that consist of thecategory of medicine and within the category medicine related to diabetes and cardiology is enlisted. Finally, the footer contains the sitemap and copyright information.

\section{2) Application Login Page}

The new user can browse the site without login but need to register from the home page to receive a verification mail and once verified the user can login using a specific username and password as shown in Fig. 6.

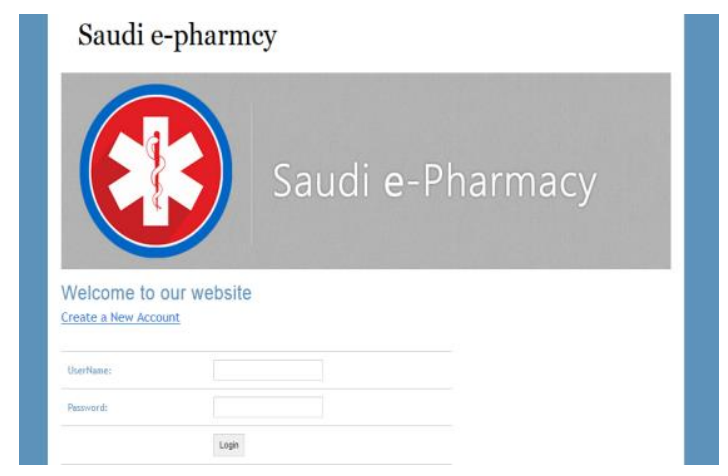

Fig 6: Illustrates Saudi e-pharmacy customer Login page

\section{3) Webpage for selecting category related medicines}

After successful login, the customer will be directed toward mycart web page if the customer has items to purchase in the cart otherwise the user will be directed toward the homepage where he can select medicine of different categories. As shown in Fig. 7the customer can select medicine related to cardiology by visiting the cardiology category of medicine from the home page. Further, the user can add medicine to the cart or delete medicine from the cart.



Fig 7: Illustrates user activity of adding medicines from category (cardiology) to cart and deleting medicine from thecart.

Fig. 8 depict the cart web page from where the customer can view/update his order details such as item quantity, price, and the total price.

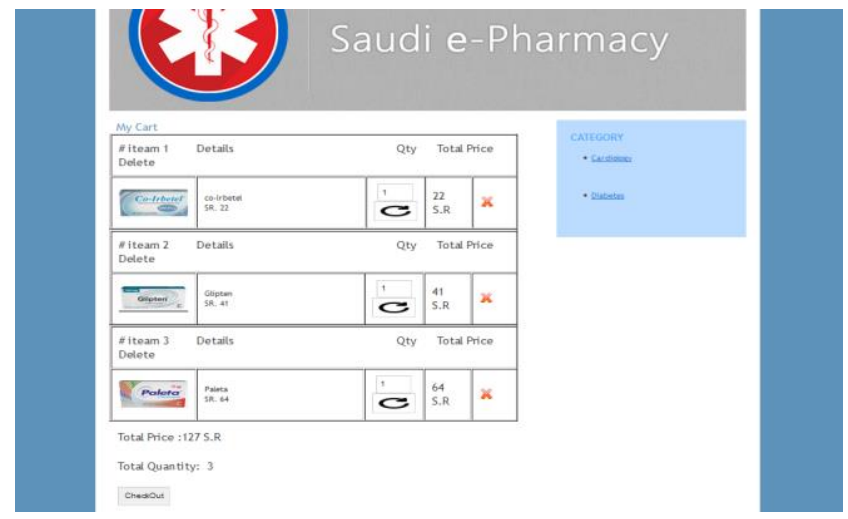

Fig 8: Illustrates the e-pharmacy cart section where the user can see and edit cart details. 


\section{4) Billing webpage of e-pharmacy}

In the billing web page, the customer can add his address and upload the e-prescription for validation as shown in Fig. 9.

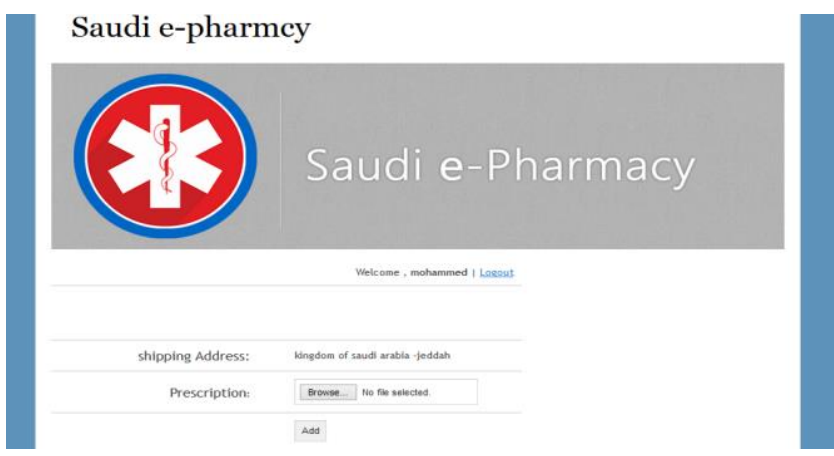

Fig 9: Illustrates Saudi e-pharmacy billing address page where user can upload Prescription

\section{5) Review Order Page}

In the review order page, the customer after reviewing his/her orderdetails can go for payment using PayPal as shown in Fig. 10.

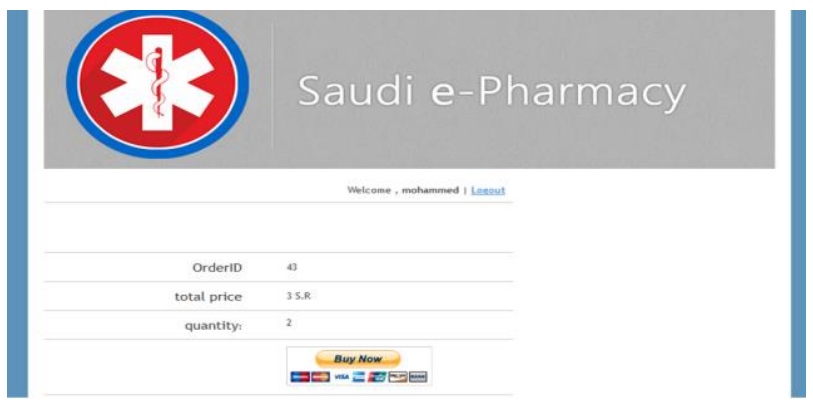

Fig 10: Illustrates the e-pharmacy payment section where the user can see and proceed for payment using any electronic payment medium.

Finally the order is placed and the payment is made using PayPal and after successful payment the customer is directed to a thank you web page where the customer can view his order number, duration for receiving his/her order and a message that he/she can view his order details from his mail or by visiting my account web page as shown in Fig. 11 and 12, respectively.

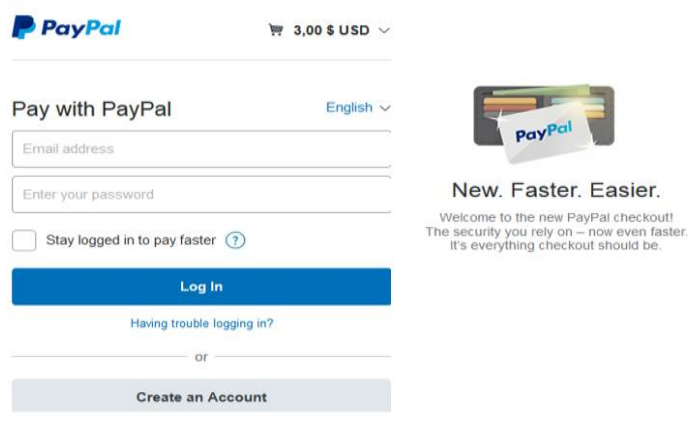

Fig 11: This is awebsitefor online payment using PayPal payment mode.

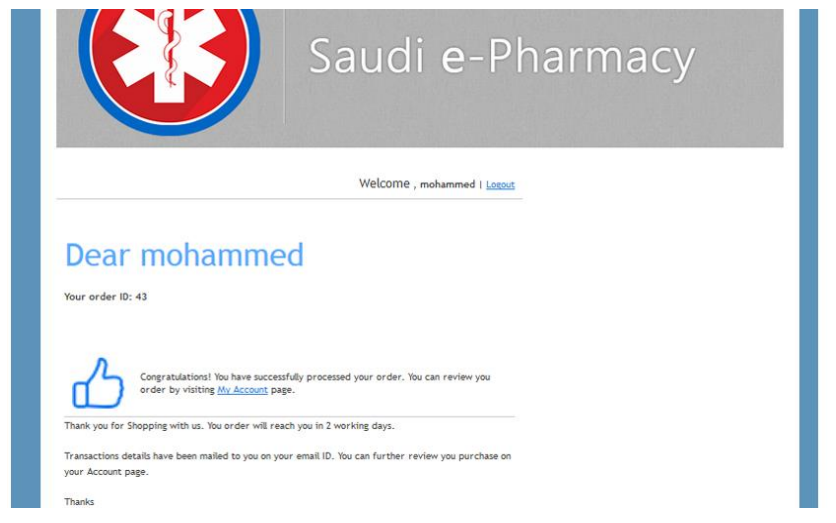

Fig 12: Illustrates the thank youpagewhen theusercompletes the payment.

\section{Testing, deployment,andmaintenance}

Black-box validation was performed to test the functionality of the application. The e-pharmacy application functionedimpeccably when tested for when tested for adifferent function such as wrong username and password for both customer and admin, misspell text-based searching of medicine etc. Further,the internal framework of the e-pharmacy application, such as checking for redundancy in the database table,database maintenance activities of admin, etc., was validated using white-box.The successful testing of the application conforms its ability to perform as per the prescribed functional and structural requirement of the application. The epharmacy prototype with will be deployed and hosted on the web soon.

\section{CONCLUSION}

The development ofthepharmacyapplicationand eprescription practices will pave the way for tapping enormousbenefits of anunexplored sector of e-commerce in Saudi Arabia. The implementation of e-prescription based purchase of medicine will decrease the prescription alteration and thus provide safety and improve the quality of service provided by to the patients or customers. Therefore, we can conclude that the online e-pharmacy platform will be used for ordering of prescription based medications for customers and will provide a platform for pharmaceutical retailers devoid of an electronic portalfor selling medicines online

\section{ACKNOWLEDGMENT}

We are thankful to our dean and the head of thedepartment for their effort for supporting and providing the necessary platform for the successful completion of this project.

\section{REFERENCES}

[1] Saudi Arabia country commercial Guide 2017 https://www.export.gov/article?id=Saudi-Arabia-ECommerce.

[2] Online shopping on the rise in Saudi Arabia: MasterCard Survey-https://newsroom.mastercard.com/mea/pressreleases/online-shopping-on-the-rise-in-saudi-arabia-mastercardsurvey.

[3] Orizio, Grazia et al. 2010. "'Save 30\% If You Buy Today'. Online Pharmacies and the Enhancement of Peripheral Thinking in Consumers." Pharmacoepidemiology and Drug Safety 19(9):970-76. Retrieved (http://dx.doi.org/10.1002/pds.2007).

[4] Orizio, Grazia, Anna Merla, Peter J. Schulz, and Umberto Gelatti. 2011. "Quality of Online Pharmacies and Websites Selling Prescription Drugs: A Systematic Review." Journal of Medical Internet Research 13(3):1-25. 
[5] Garrett, S., \& Skevington, P. (2013). An introduction to electronic commerce. BT Technology Journal, 2(4), 190-193. http://doi.org/10.1038/183777b

[6] Awais, M., \& Samin, T. (2012). Advanced SWOT Analysis of E-Commerce. International Journal of Computer Science Issues, 9(2), 569-574. Retrieved from http://ijcsi.org/papers/IJCSI-9-22-569-574.pdf

[7] I. Sommerville, Software Engineering. 2010.

[8] Jacobson, Ivar; Spence, Ian; Bittner, Kurt (December 2011). "Use Case 2.0: The Guide to Succeeding with Use Cases". Ivar Jacobson International. Retrieved 2014-05-05.

[9] Davis, Ian. "What Are The Benefits of MVC?". Internet Alchemy. Retrieved 2016-11-29.

[10] "What is MySQL?". MySQL 5.1 Reference Manual. Oracle. Retrieved 17 September 2012. The official way to pronounce "MySQL" is "My Ess Que Ell" (not "my sequel")
[11] Flanagan, David. JavaScript - The definitive guide (6 ed.). p. 1. JavaScript is part of the triad of technologies that all Web developers must learn: HTML to specify the content of web pages, CSS to specify the presentation of web pages, and JavaScript to specify the behavior of web pages.

[12] Lerdorf, Rasmus (2007-04-26). "PHP on Hormones - history of PHP presentation by Rasmus Lerdorf gave at the MySQL Conference in Santa Clara, California". The Conversations Network. Retrieved 2009-12-11.

[13] Jerry Gao; H.-S. J. Tsao; Ye Wu (2003). Testing and Quality Assurance for Component-based Software. Artech House. pp. 170-. ISBN 978-1-58053-735-3.

[14] Ehmer Khan, Mohd (July 2011). "Different Approaches to White Box Testing Technique for Finding Errors" (PDF). International Journal of Software Engineering and Its Applications. 5: 1-6. 